\title{
Ecological management technology for coarse sediment area in the Yellow River Basin
}

\author{
Zhenzhou Shen ${ }^{1}$, Binbin $\mathrm{Li}^{* 2}$, Han Deqiang*3, Zhang Hong ${ }^{3}$, Shiping Cheng ${ }^{4}$, Liting $\mathrm{Hou}^{5}, \mathrm{Cao} \mathrm{Li}^{3}$, Chang Yideng ${ }^{3}$ \\ ${ }^{1}$ Yellow River Institute of Hydraulic Research, Zhengzhou, 450003, China \\ ${ }^{2}$ Water and Soil Conservation Monitoring Center of Ministry of Water Resources, Beijing 100053,China \\ ${ }^{3}$ Xinjiang Kurgan Water Conservancy Project Construction Administration, Kashi, Xinjiang, China 848400 \\ ${ }^{4}$ Pingdingshan college. Pingdingshan, 450051, China \\ ${ }^{5}$ Yellow River Watershed Ecological Water Conservancy Engineering Research Center, Yellow River Conservancy Technical College, \\ Kaifeng, 450003, China;
}

\begin{abstract}
Across wide range of research survey of the Pisha stone area landform, erosion and vegetation characteristics, on the basis of conducting the Pisha stone under different conditions of underlying surface erosion characteristics of the test, at the same time the wide range of investigation, access, summarizes the local government and the management experience and management pattern of the masses, summarizes the research of Yulin Pisha stone areas covered sand erosion governance approach and the prevention and control technology.
\end{abstract}

\section{Introduction}

Pisha stone areas is located in Jin-Shan-Meng of border area, covers an area of $16.7 \times 10^{3}$ square kilometers, accounts for only $7.1 \%$ of the area of the loess plateau soil and water loss, but the sediment into the Yellow River is 350 million tons per year, about 280 million tons, the coarse sand is the severe erosion center of the loess plateau and environment of the worst areas, also is the main source of the Yellow River sand, coarse sand area. Arsenic sandstone is the common name of sandy and gravel-type calcous coarse bone soil and mud-shale calcous coarse bone soil. Its loose structure is easily eroded by freezing and thawing. Generally, it is eroded by $5-10 \mathrm{~cm}$ every year. Due to the fragile ecological environment, barren land and degraded grassland in the arsenic sandstone area, the local economic development is greatly restricted.

In order to change the ecological environment of Pisha sandstone area, reduce the amount of yellow sand and quicken the pace of local people to get rich, the state officially carried out the "Ecological Project of Seabuckthorn in Jin-Shan-Meng Pisha Sandstone Area" in 1998. It covers an area of 20,000 square kilometers ${ }^{[1-}$ ${ }^{3]}$. The project is planned to spend 13 years $(1998-2010)$ to build a new sea buckthorn forest of 30 million mu and control the soil erosion area of 20,000 square kilometers in the arsenic sandstone area.After the completion of the project ${ }^{[4-8]}, 170$ million tons of yellow sediment will be reduced every year, including 140 million tons of coarse sand, and the ecological environment and agricultural production conditions in the project area will be significantly improved. 


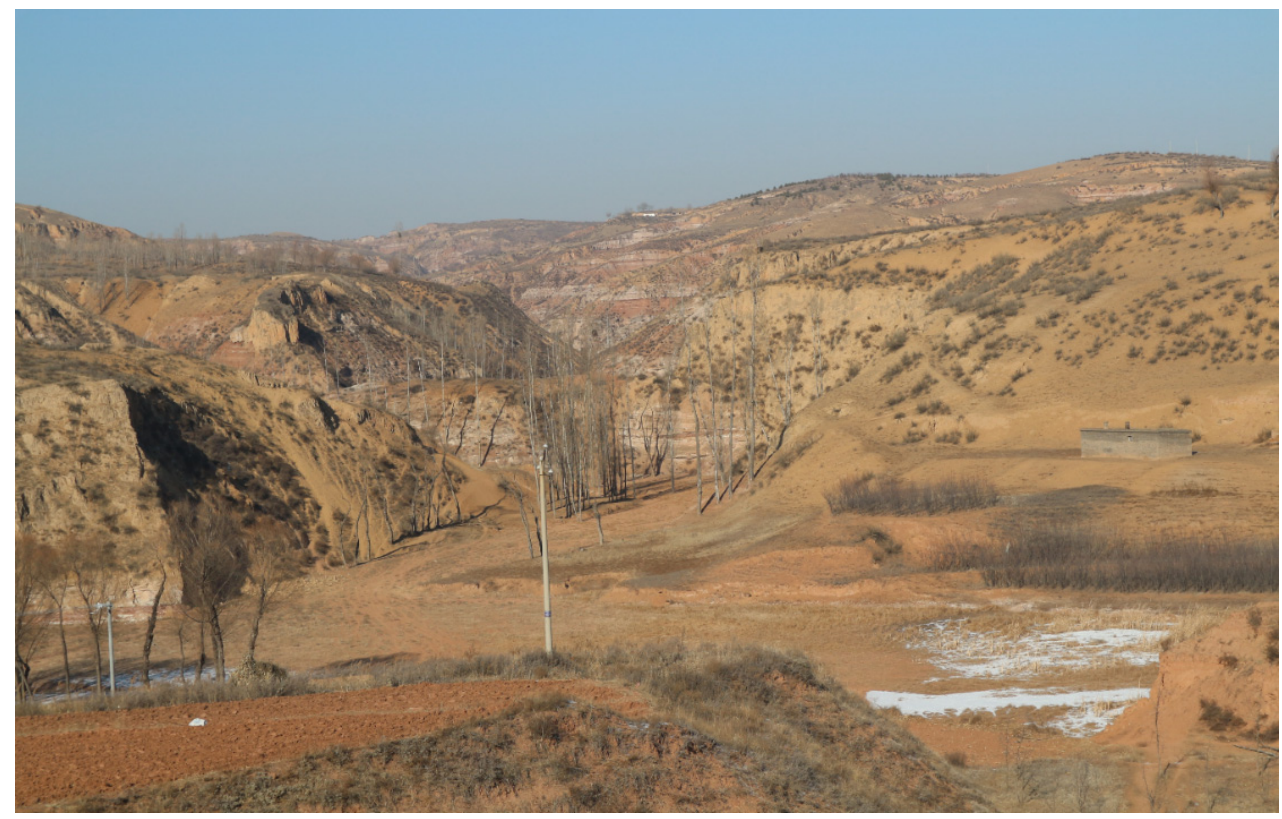

Fig.1 Topographical features in sand-covered Pisha stone areas

\section{Results and analysis}

On the basis of surveying the topographic and geomorphic features, erosion and vegetation characteristics of the sandstone in a large scale, the erosion characteristics of the sandstone under different underlying surface conditions were carried out.

\subsection{Suggestions on vegetation construction in Pisha Sandstone Area}

\subsubsection{Breakthrough of biological treatment in Pisha Sandstone Area}

According to the growth performance of seabuckthorn, seabuckthorn can be used as a breakthrough for biological control in soft sandstone area, especially suitable for planting on the sand covered soft sandstone with serious soil erosion, so as to give full play to its excellent protection effect. In the long run, planting Hippophae rhamnoides should be combined with the characteristics of this area. For the gentle area, wide-band planting should be adopted as far as possible, and the spacing between plants and rows should be $1-24 \mathrm{~cm} \times 6-10 \mathrm{~cm}$, so as to facilitate grazing. It is strongly advocated to build Seabuckthorn mixed forest to create a suitable environment for the growth and development of other plants while giving full play to the benefits of water and soil conservation of seabuckthorn and improving the soil function, so as to promote the development of vegetation in the region to a benign succession direction. In addition, we should strengthen the research and development of seabuckthorn varieties to improve its economic value.

\subsubsection{Planting Caragana korshinskii on soft sandstone of GAISHA}

Caragana korshinskii is resistant to drought and barren, and can adapt to a wide range of soil conditions. It grows well in various soil conditions in Pisha Sandstone Area, especially in loose sandy soil. Therefore, it should be popularized and planted in the soft sandstone area. In order to make full use of the grazing value of Caragana korshinskii, rotation grazing, timely stubble leveling and rational utilization should be advocated. The low yield sandy grassland can be improved by replanting or replanting Caragana korshinskii to improve its utilization value.

\subsubsection{Pinus tabulaeformis and Hippophae rhamnoides can be used as the main tree species for afforestation in Pisha Sandstone Area}

The results show that Pinus tabulaeformis can grow normally on the Pisha Sandstone slope with an average annual height of $20-30 \mathrm{~cm}$, and Hippophae rhamnoides can grow well on both slope and gully with an average annual height of $20-50 \mathrm{~cm}$. Therefore, Pinus tabulaeformis and Hippophae rhamnoides are the main tree species for afforestation in Pisha Sandstone Area, but Pinus tabulaeformis is only suitable for afforestation on gentle slope of Pisha Sandstone.

\section{2 comprehensive treatment and development of Pisha Sandstone Area}

According to the specific situation of Pisha Sandstone Area and the needs of economic development, the general idea of governance and development in this area is as follows: Taking the construction of channel dam system as the breakthrough point, strengthening the construction of channel dam system based on the key project of gully governance; according to local conditions, actively promoting the construction of forest and grass vegetation, returning farmland to forest and grass, implementing large-scale closure and governance, giving full play to the self-healing function of ecosystem. 


\subsubsection{Implement ecological restoration and seabuckthorn resources construction}

In addition to the construction of energy and heavy chemical industry base and the acceleration of urbanization process, the growth trend of rural population has gradually slowed down. Although the spatial and temporal distribution of rainfall is uneven, the annual average rainfall is about $400 \mathrm{~mm}$. The implementation of ecological restoration in this area will be one of the measures with the fastest speed, the best benefit and the lowest cost in ecological construction. At the same time, seabuckthorn resources construction was carried out in the area, horizontal terraces were built on the gentle slope land near the village, near the road and with irrigation conditions, and water cellars were built in villages and courtyards to solve the problem of drinking water for human and livestock and develop courtyard economy.

\subsubsection{The tributary dam system will be constructed with the large and medium sized mud retaining reservoir as the framework and the warping dam as the main body}

According to the research results of dam system engineering layout, in general, the proportion of backbone dam and warping dam is between 1:2 and 1:5, while the proportion of backbone dam is too large in Pisha Sandstone dam system, and the proportion of medium and small warping dams is too small. Of course, the emergence of this situation is related to the development stage of dam system. These dam systems have only developed from the first stage to the mature dam system. Medium and small warping dams are still in the initial stage, and they are the main body of dam system construction in the future. Small and medium sized warping dams should be built in the branch furrows with a drainage area of less than $3 \mathrm{~km} 2$, mainly for retaining silt and silting up land and developing basic farmland; in the branch furrows with a drainage area of more than $3 \mathrm{~km} 2$, key gully control projects should be built to ensure the safe production of downstream dam land; Large and medium sized mud storage tanks should be built in large branch ditches or main streams which are difficult to control and have good conditions; reservoirs and ponds should be set up in ditches with water sources to provide water for urban domestic and industrial water and agricultural irrigation.

\subsubsection{The integrated technology of plant "flexible dam" and warping dam is adopted to realize coarse and fine sediment discharge}

The seabuckthorn plant "flexible dam" is arranged near the warping dam body, the upstream tail end and the upper part of the spillway inlet. The main body of the project is the plant "flexible dam", and the main components of the basic farmland at the bottom of the gully are the gully check dam, "artificial wetland" and "artificial beach". Relying on the backbone dam and guaranteed by the micro reservoir, Zhimao gully is formed to intercept coarse sand, "artificial beach" and channel dam to intercept fine sand, and "artificial wetland" and channel dam are formed between dams to increase the inflow of natural runoff. The micro reservoir can store all the remaining runoff, so as to slow down the flood, retain the coarse sediment, discharge the flood into the river, realize the coarse deposition and fine discharge, improve the water and sediment conditions and sediment composition into the downstream river, and maintain the ecological function of the river.

\subsubsection{Erosion prevention and control technology in soft sandstone area covered with sand}

In the area covered by sand, the mixed forest or shrubbery of tree and shrub belt is mainly arranged on the ridge, hill and slope, and the high-quality forage grass is intercropped, which can not only prevent wind and fix sand, but also be used as the animal husbandry base. Caragana korshinskii was planted in fixed sand dunes perpendicular to the main wind direction, grass was planted between the belts, and contour planting was carried out in water erosion area. In mobile and semi mobile sand areas, Salix psammophila is planted in belts perpendicular to the main wind direction, and yangchai and Astragalus adsurgens are planted between belts. Poplar and willow are the main soil and gully with good water condition, and timber forest should be developed.

\subsubsection{Increase the management and protection work after construction, reduce man-made damage and consolidate the treatment results}

According to the survey, the conservation rate of the soil and water conservation measures currently adopted is low, especially the vegetation measures. Due to climate factors and human destruction, the survival rate of vegetation is low. Therefore, it is necessary to strengthen the post construction management and protection to consolidate the control effect. In the aspect of management and protection, we should further strengthen the publicity of laws and regulations related to soil and water conservation, improve the management and protection institutions, make clear division of labor, clear responsibilities and rights, regularly check the control results, and take timely remedial measures. Ensure that all measures are effective.

\subsubsection{Increase capital investment, strengthen environmental management}

The construction of soil and water conservation has a large investment and slow effect, but it is a grand project benefiting future generations. For the control areas, most of them are in the rural areas with poor living conditions. Due to the low living standard, some soil and water conservation measures are difficult to implement, or are damaged after the implementation, resulting in the lack of stamina of this work. Therefore, it is necessary to pay enough attention to the soil and water conservation work in terms of funds and publicity Only in this way can soil and water conservation measures be avoided from beginning to end. 


\section{Conclusion}

Across wide range of research survey of the Pisha stone landform, erosion and vegetation characteristics, on the basis of conducting the Pisha stone under different conditions of underlying surface erosion characteristics of the test.

At the same time wide range of investigation, access, summarizes the local government and the management experience and management pattern of the masses, summarizes the research of Yulin Pisha stone areas covered sand erosion governance approach and the control technology.

\section{Acknowledgements}

This work is funded by the State Key Research Development Program of China (Grant No. 2017YFC0504503), and the National Natural Science Foundation of China (Grant No. 41201267).

\section{REFERENCES}

1. Xi jinping. Speech at the symposium on ecological protection and high-quality development of the Yellow River basin [J]. Truth seeking, 209, 20:1-2

2. Wang Yuanchang, $\mathrm{Wu}$ Yonghong, Kou Quan et al. Definition of arsenic rock zone borderline and its classification $[\mathrm{J}]$. Science of Soil and Water Conservation, 2007, 5(1):14-18

3. YAO Wenyi, SHI Mingli, WU Zhiren. Management Technology and Demonstration Effect on TwoDimensional Configuration in Pisha Sandstone Area[J]. YELLOW RIVER,2016,38(6):1-7

4. Liu Baoyuan, Liu xiaoyan, Yang qinke, et al. Investigation report on comprehensive control of soil and water loss in small watershed [J]. Soil and water conservation bulletin, 2017,37 (4)

5. Zheng fenli, Xu Ximeng QinChao. Gully erosion process research progress [J]. Journal of agricultural machinery, 2016, (8) : 48-59116.

6. Shen Zhenzhou, Liu Puling, Xie Yongsheng et al. Transformation of erosion types on loess slope by REE tracking [J], Journal of Rare Earths, 2007, 25 (4): 67-73.

7. Shen Zhenzhou, Yao Wenyi, Li Mian et al, Influence factors of runoff energy consumption and denudation amount on loess slope $[\mathrm{J}]$. Science of Soil and Water Conservation, 2009, 7 (6): 9-13.

8. SHEN Zhenzhou,YAO Wenyi, L I Mian, et al. Study on Correlativity Between Erosion and Seeping Under Different Horizon[J], Journal of Soil and Water Conservation, 2008, 22 (5): 43-46. 